\title{
Incidence and Age Distribution of Hospitalized Presumptive and Possible Abusive Head Trauma of Children Under 12 Months Old in Japan
}

\author{
Yui Yamaoka ${ }^{1,2}$, Takeo Fujiwara ${ }^{3}$, Yoshihisa Fujino ${ }^{4}$, Shinya Matsuda ${ }^{5,6}$, and Kiyohide Fushimi ${ }^{7}$ \\ ${ }^{1}$ Department of Health Services Research, University of Tsukuba, Ibaraki, Japan \\ ${ }^{2}$ Center on Child Abuse and Neglect, University of Oklahoma Health Sciences Center, Oklahoma, USA \\ ${ }^{3}$ Department of Global Health Promotion, Tokyo Medical and Dental University, Tokyo, Japan \\ ${ }^{4}$ Department of Environmental Epidemiology, Institute of Industrial Ecological Sciences, \\ University of Occupational and Environmental Health, Fukuoka, Japan \\ ${ }^{5}$ Department of Preventive Medicine and Community Health, School of Medicine, University of Occupational and Environmental Health, Fukuoka, Japan \\ ${ }^{6}$ Data Science Center of Occupational Health, University of Occupational and Environmental Health, Fukuoka, Japan \\ ${ }^{7}$ Department of Health Policy and Informatics, Tokyo Medical and Dental University, Tokyo, Japan
}

Received May 7, 2018; accepted January 7, 2019; released online February 2, 2019

\begin{abstract}
Background: Abusive head trauma (AHT) is the leading cause of fatal maltreatment among young children. The incidence of AHT in Japan, however, remains unknown. This study examined the incidence and distribution of age in months among young children under 12 months of age hospitalized with intracranial injury in Japan.

Methods: We conducted a multicenter cross-sectional study of children under 36 months old admitted with intracranial injury to hospitals that employed the Diagnostic Procedure Combination (DPC) payment system between 2010 and 2013. Presumptive and possible AHT were defined using the combination of ICD-10 codes modified from the coding system recommended by the United States Centers for Disease Control and Prevention.

Results: The average incidence was 7.2 (95\% confidence interval [CI], 7.18-7.26) for presumptive and 41.7 (95\% CI, 41.7-41.8) for possible AHT per 100,000 children less than 12 months old from 2010 to 2013 . The distributions of age in months for both presumptive AHT and possible AHT had peaks at around 2 and 8 months.

Conclusions: This is the first study to report the incidence of hospitalized children with presumptive and possible AHT using population-based data. Further datasets are needed to evaluate the incidence and specific preventive strategies to prevent AHT in infants during the months of highest risk.
\end{abstract}

Key words: abusive head trauma; intracranial injury; ICD-10; incidence; Japan

Copyright ( $\odot 2019$ Yui Yamaoka et al. This is an open access article distributed under the terms of Creative Commons Attribution License, which permits unrestricted use, distribution, and reproduction in any medium, provided the original author and source are credited.

\section{INTRODUCTION}

Abusive head trauma (AHT), or shaken baby syndrome (SBS), is the leading cause of death due to child maltreatment ${ }^{1,2}$ and may lead to neurological sequelae. ${ }^{3}$ To evaluate prevention strategy, the incidence of AHT needs to be reported repeatedly. Using active surveillance, the incidence of admitted AHT children (per 100,000 children) was reported from 12.5 to 38.8 in several Western countries, such as United States (children under 1 year old), ${ }^{4,5}$ United Kingdom (children under 2 years old), ${ }^{6}$ Australia (children under 2 years old), ${ }^{7}$ or New Zealand (children under 2 years old). ${ }^{8}$ These active surveillance systems are expensive, however, and recent studies have demonstrated the validity of passive surveillance systems using the algorithm of International Classification of Diseases, Tenth Revision (ICD-10) codes for hospitalization records. ${ }^{9,10}$
Although the epidemiology of AHT shares similarity among countries, it has not been sufficiently examined in Japan. The only "self-reported" prevalence for shaking was reported as 3.9\% in a population-based study, ${ }^{11}$ which was similar to that in Western countries. However, there are few studies that examine the incidence of AHT using hospitalization records in Japan.

Japan has a unique health care system and medical facilities. Universal health coverage was established in $1958,{ }^{12}$ and everyone is insured and has unlimited access to all health care facilities at a relatively low cost. In addition, Japan has one of the highest penetration rate of computed tomography (CT) and magnetic resonance imaging (MRI) in developed countries. ${ }^{13}$ The number of CT and MRI scans was approximately three- to fourfold higher than the average for Organization for Economic Cooperation and Development (OECD) countries. Thus, Japanese hospitalization records may have the potential capacity to detect 
Table 1. International Classification of Diseases, $10^{\text {th }}$ version (ICD-10) codes to identify presumptive and possible abusive head trauma (AHT)

\begin{tabular}{|c|c|c|}
\hline ICD-10 Code & & Description \\
\hline \multirow{2}{*}{ 1. Intracranial injury codes } & S06.0-S06.9 & Intracranial injury \\
\hline & T90.5 & Sequelae of intracranial injury classifiable to S06 \\
\hline \multirow{4}{*}{ 2. Intentional injury codes } & T74.1, T74.8, T74.9 & Physical abuse; Other maltreatment syndromes; \\
\hline & $174.1,174.0,174.9$ & Maltreatment syndrome, unspecified \\
\hline & Y00, Y01, Y04, Y08, Y09 & Assault \\
\hline & Y07.0, Y07.1, Y07.2, Y07.3, Y07.8, Y07.9 & Other maltreatment \\
\hline 3. Retinal hemorrhage & H35.6 & Retinal hemorrhage \\
\hline \multirow{3}{*}{ 4. Unintentional injury codes } & V01-V99 & Transport accident \\
\hline & W00-X59 & Other externa cause of accidental injury (including falls) \\
\hline & Y85-Y86 & Sequelae of transport accident; Sequelae of other accidents \\
\hline \multirow{6}{*}{ 5. Excluding codes } & D65-69 & Coagulation defects, purpura and other hemorrhagic conditions \\
\hline & E56.1 & Deficiency of vitamin $\mathrm{K}$ \\
\hline & P53 & Hemorrhagic disease of the fetus and newborn \\
\hline & P10-P15 & Birth trauma \\
\hline & P52.8 & Other intracranial (nontraumatic) hemorrhages of the fetus and newborn \\
\hline & P52.9 & Intracranial (nontraumatic) hemorrhage of the fetus and newborn, unspecified \\
\hline
\end{tabular}

Presumptive AHT cases were [Intracranial injury codes] AND [Intentional injury codes AND/OR Retinal hemorrhage] WITHOUT [Excluding codes].

Possible AHT cases were presumptive AHT cases plus [Intracranial injury code] WITHOUT [Unintentional injury codes AND/OR Excluding codes].

intracranial injury among young children. A nationwide study investigating the incidence of admitted AHT is necessary to confirm the similarity of the incidence of AHT regardless of health care system or medical equipment.

Furthermore, Fujiwara et $\mathrm{al}^{14}$ reported that the prevalence of AHT diagnosed with CT showed two peaks: around 2 to 4 months and around 7 to 9 months. The second peak around 7 to 9 months is not clearly reported in other countries (ie, United States, ${ }^{15-17}$ Scotland, ${ }^{18}$ or China ${ }^{19}$ ), and the mechanisms and preventive strategies for later infancy remain unknown. Since study subjects in the previous study are limited to a single national central hospital, ${ }^{14}$ the distribution of ages in month for AHT using nationwide samples with hospital-based data need to be confirmed. Therefore, this study examined the incidence of hospitalized AHT for infants under 12 months old and described the distribution of ages in month for AHT children under 36 months old using hospital-based medical claim data.

\section{METHODS}

\section{Data source}

This study utilized the Diagnosis Procedure Combination (DPC) database, which includes patient discharge and administrative claims. ${ }^{20}$ The DPC database does not include cases due to motor vehicle accidents because administrative claims do not cover such. All advanced treatment hospitals have been directed to adopt the DPC in 2003, and other general hospitals have adopted it voluntarily. Advanced treatment hospitals have capabilities to provide advanced medical care and technologies with $400+$ beds and $16+$ clinical departments. The number of participating hospitals increased from 82 hospitals with 68,982 inpatient beds in 2003 to 1,585 hospitals with 492,206 inpatient beds in 2014 . This number includes more than half of all general hospital beds nationwide. $^{21}$ The DPC database includes the demographics of patients, diagnosis, and comorbidities using the ICD-10 codes, treatments and surgeries, length of stay, and discharge status. ${ }^{22,23}$ Physicians in charge must code the diagnosis according to medical records, and medical clerks or licensed medical information managers record all drugs, devices, and procedures during the admission. ${ }^{24}$

\section{Identifying suspected AHT}

This study targeted children under 36 months old who were admitted to a hospital in the DPC database due to intracranial injury from 2010 through 2013. We extracted children with suspected AHT who had at least one diagnosis from multiple categories of disease diagnosis ("main diagnosis," "admissionprecipitating diagnosis," "most resource-consuming diagnosis," "second most resource-consuming diagnosis," "comorbidities present at time of admission," and "conditions arising after admission"). ${ }^{22,23}$ We defined AHT based on certainty of AHT, that is, "presumptive AHT" definitions following the recommendation by the United States Centers for Disease Control and Prevention (CDC) $)^{9,25}$ and "possible AHT" definition instead of "probable AHT" used in previous studies ${ }^{5,9,10,27}$ as shown in Table 1. We decided to use the term "possible" AHT in this study because DPC data did not clearly have codes for unintentional injuries. The considerations for these differences among definitions are mentioned in the discussion section. Presumptive AHT represented children who had intracranial injuries (ICD-10 intracranial injury code: S06.0-S06.9, T90.5) and either an intentional injury codes (physical abuse: T74.1, other maltreatment syndromes, T74.8, maltreatment syndrome, unspecified, T74.9, assault: Y00, Y01, Y04, Y08, Y09; other maltreatment; Y07.0, Y07.1, Y07.2, Y07.3, Y07.8, Y07.9) or retinal hemorrhage (H356), without any kind of exclusion criteria for plausible causes including coagulation defects, purpura and other hemorrhagic conditions (D65-D69), deficiency of vitamin K (E56.1), birth trauma (P10-P15), intracranial nontraumatic hemorrhage of the fetus and newborn (P52), and hemorrhagic disease of the fetus and newborn (P53). Possible AHT included, in addition to presumptive cases, children who had intracranial injury (S06.0-S06.9, T90.5) without unintentional injury code (transport accident [V01-V99], other external causes of accidental injury including falls [W00-X59], sequelae of transport accident [Y85], and sequelae of other accidents [Y86]), applying the above excluding criteria (eg, coagulation defects). If the children were admitted multiple times in the same year, we selected one admission due to presumptive AHT; poorer outcome at discharge, if multiple diagnoses were the same (presumptive or possible); or a younger age, if the other conditions were the same. 


\section{Analysis}

We described each annual number and the proportion of total admissions, intracranial injury admissions, possible and presumptive AHT at the age of 0-11, 12-23, and 24-35 months old. The number of hospitals that have adopted the DPC system increased year by year, and the number of infants less than 12 months old admitted to DPC hospitals increased from 147,776 in 2010 to $176,956,206,681$, and 203,301 in 2011, 2012, and 2013, respectively. Therefore, the number of infants as the denominator was adjusted by the number of births each year multiplied by the ratio of the increase of admissions to DPC hospitals in each year compared with that of in 2013. In addition, we took into account the coverage rate of the DPC system among hospitals nationwide. The formula for calculating incidence in each year for children under 12 months old is:

$$
\begin{aligned}
& \text { Incidence of presumptive AHT (y) } \\
& \qquad=\frac{\text { number of presumptive AHT }(y)}{\text { number of infant population in Japan }(y) \times \frac{\text { total number of infant cases in DPC }(y)}{\text { total number of infant cases in DPC in } 2013}} \times \frac{1}{Z}
\end{aligned}
$$

here, (y) represents each year, and $\mathrm{Z}$ represents the proportion of DPC introduced hospitals among hospitals having capabilities to provide advanced pediatric care, such as advanced treatment hospitals, children's hospitals, and related institutions.

In addition to 83 advanced treatment hospitals, there were 36 children's hospitals, which belonged to the Japanese Association of Children's Hospitals and Related Institutions (JACHRI). These hospitals can provide comprehensive pediatric care with $100+$ pediatric beds, 20+ pediatricians per 100 beds, and CT/MRI facilities. Twenty-five hospitals $(69.4 \%$ of children's hospitals and related institutions) and all 83 advanced treatment hospitals employed the DPC payment system. We assumed that all the AHT admission cases would be captured in these advanced treatment hospital and JACHRI. In total, 108 (ie, 83 plus 25) out of 119 (ie, 83 plus 36) advanced treatment hospitals and children hospitals and related institutions were covered in the DPC payment system $(90.8 \%)$, which is the same during the observed period (ie 2010-2013). Therefore, we used the coverage rate (Z) as $90.8 \%$ for all years.

Next, we examined the differences between presumptive and possible AHT cases among children under 36 months old. We compared demographics (ie, sex, age), clinical conditions (ie, Japan Coma Scale [JCS], duration of admission, transfer from other hospital, use of ambulance), outcome at discharge with six categories (ie, cured, improved, remission, remain unchanged, exacerbation, and death), and admission cost. We dichotomized outcomes into poor (remain unchanged, exacerbation, or death) and better (cured, improved, or remission) outcomes. Under the DPC payment system, the total admission cost, which is based on diagnosis group, includes basic hospital stays and any treatments during the admission, such as laboratory tests, medications, injections, and surgical procedures. In addition, for sensitivity analysis, the month-age distribution of epidural hemorrhage (EDH; S06.4) was compared between presumptive AHT, possible AHT and possible AHT excluding EDH, because EDH is more likely to occur after falls. ${ }^{26}$ Lastly, we described the distribution of age in months for presumptive AHT, possible AHT, and possible AHT excluding EDH. We used STATA MP, version 14.0 (Stata Corp LP, College Station, TX, USA). The ethical review board at Tokyo Medical and Dental University approved this study.

\section{RESULTS}

Table 2 shows the number of admissions with presumptive and possible AHT among children under 36 months old from 2010 through 2013. The number of total admissions of children increased from 2010 through 2013 because the number of hospitals enrolled in the DPC database rose year by year. Among presumptive AHT admissions, the majority were infants (61.4-81.6\%), and the proportion of older children was relatively small. Among possible AHT, almost half of them were infants; however, the proportion of children aged 24-35 months old increased from $23.2 \%$ to $25.5 \%$. Among possible AHT, $11.1 \%$ to $13.5 \%$ were presumptive AHT.

Table 3 and Figure 1 shows the incidence of presumptive and possible AHT per 100,000 infants, adjusted by the changes in the number of admissions in DPC hospitals from 2010 through 2013 and the coverage of DPC system among hospitals where can provide advanced pediatric treatment. The overall incidence was 7.22 (95\% confidence interval [CI], 7.18-7.26) per 100,000 infants for presumptive AHT, 41.7 (95\% CI, 41.7-41.8) per 100,000 infants for possible AHT. The incidence for presumptive AHT increased $(P$ for trend $=0.042$ ), but the incidence of possible AHT was stable between from 2010 through 2013 ( $P$ for trend $=0.950$ ).

Table 4 shows the differences between children less than 36 months age admitted with presumptive and possible AHT. Children with presumptive AHT were younger, had a poorer JCS score, longer hospitalization, were more often transferred from another hospital, more often used an ambulance, and showed poorer outcome at discharge than children with possible AHT. The proportion of EDH was higher among possible AHT cases than in presumptive AHT (18.8\% vs $6.8 \%)$. The medians of the admission costs were JPY 754,000 (approximately USD 7,540) and JPY 240,000 (approximately USD 2,400) for presumptive and possible AHT per case, respectively.

Figure 2 shows the distribution of age in months for presumptive AHT, possible AHT, and possible AHT excluding EDH. For both presumptive and possible AHT, we observed two peaks, at 1-3 and 7-9 months old. Further, possible AHT excluding EDH cases also had two peaks at 1-3 and 7-9 months old. After 12 months old, the number of both presumptive and possible AHT cases became relatively stable.

\section{DISCUSSION}

This is the first study to describe the incidence of presumptive and possible AHT using ICD-10 codes for admissions in Japan. The incidences were 7.2 for presumptive AHT and 41.7 for possible AHT per 100,000 infants less than 12 months old from 2010 
Table 2. Admissions with presumptive and possible abusive head trauma (AHT) among children under 36 months old in $2010-2013$

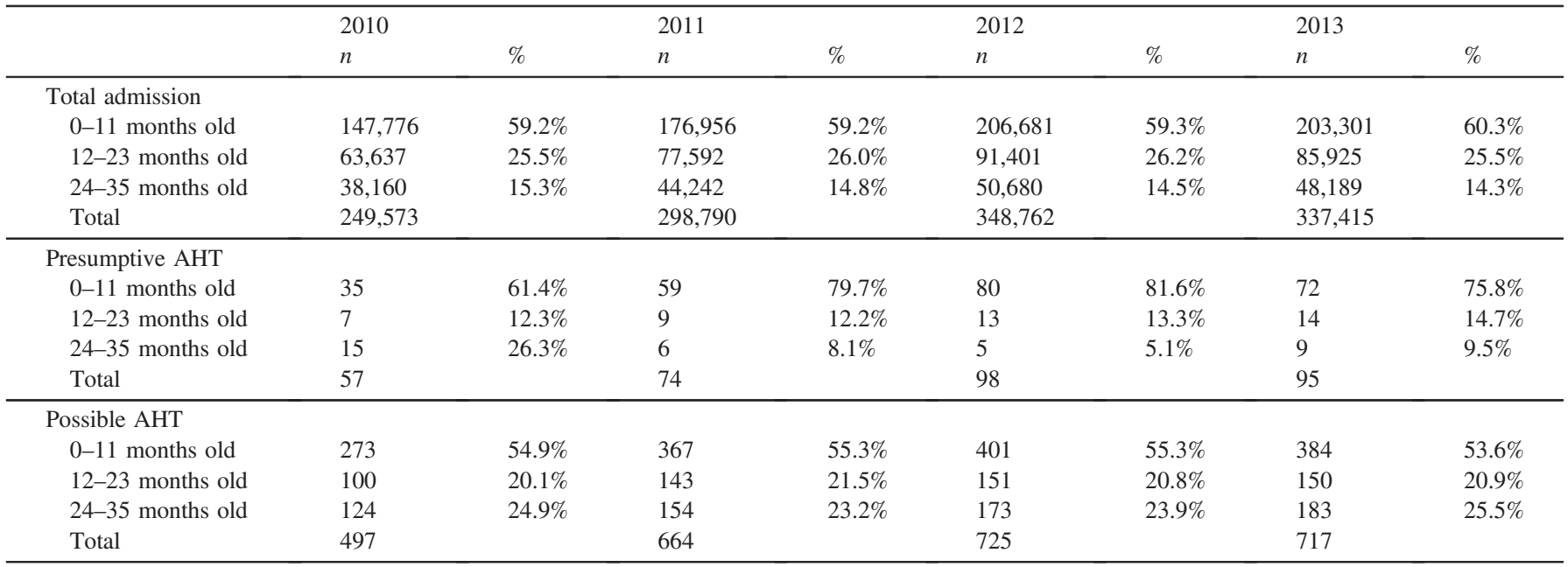

Table 3. Incidence of presumptive and possible abusive head trauma (AHT) in children under 12 months of age

\begin{tabular}{|c|c|c|c|c|c|}
\hline Year & 2010 & 2011 & 2012 & 2013 & $\begin{array}{l}\text { Total } \\
(2010-2013)\end{array}$ \\
\hline Number of Presumptive AHT $(a)$ & 35 & 59 & 80 & 72 & 246 \\
\hline Number of Possible AHT $(a)$ & 273 & 367 & 401 & 384 & 1,425 \\
\hline Infant population $(c)$ & $1,037,633$ & $1,060,000$ & $1,032,000$ & $1,030,000$ & $4,159,633$ \\
\hline Number of admissions in DPC hospitals $(d)$ & 147,776 & 176,956 & 206,681 & 203,301 & 734,714 \\
\hline $\begin{array}{l}\text { Ratio to the number of admissions in DPC hospitals compared } \\
\text { to that of } 2013(d / e)\end{array}$ & 0.73 & 0.87 & 1.02 & 1.00 & \\
\hline Adjusted infant population $\left(b=c^{*} d / e\right)$ & 754,238 & 922,639 & $1,049,158$ & $1,030,000$ & $3,756,035$ \\
\hline Incidence of presumptive AHT $\left(/ \mathbf{1 0 0 , 0 0 0}\right.$ infants) $\left(a / b^{*} 1 / f\right)$ & 5.1 & 7.0 & 8.4 & 7.7 & 7.2 \\
\hline Incidence of possible AHT $(/ \mathbf{1 0 0 , 0 0 0}$ infants $)\left(a / b^{*} 1 / f\right)$ & 39.8 & 43.8 & 42.0 & 41.0 & 41.7 \\
\hline
\end{tabular}

DPC, Diagnosis Procedure Combination.

a. $\mathrm{N}$ of AHT (year): Number of presumptive AHT of infants in each year.

$b$. Adjusted $\mathrm{N}$ of infant (year): Number of infant population each year adjusted by the increase of DPC hospital admissions compared to 2013.

c. $\mathrm{N}$ of infant (year): Number of infant population in each year.

d. $\mathrm{N}$ of DPC (year): Number of total infant admissions in each year.

$e$. N of DPC (2013): Number of total infant admissions in 2013.

$f$. Coverage of DPC system among advanced treatment hospitals and children hospitals (90.8\%).

$(/ 100,000$ infants)

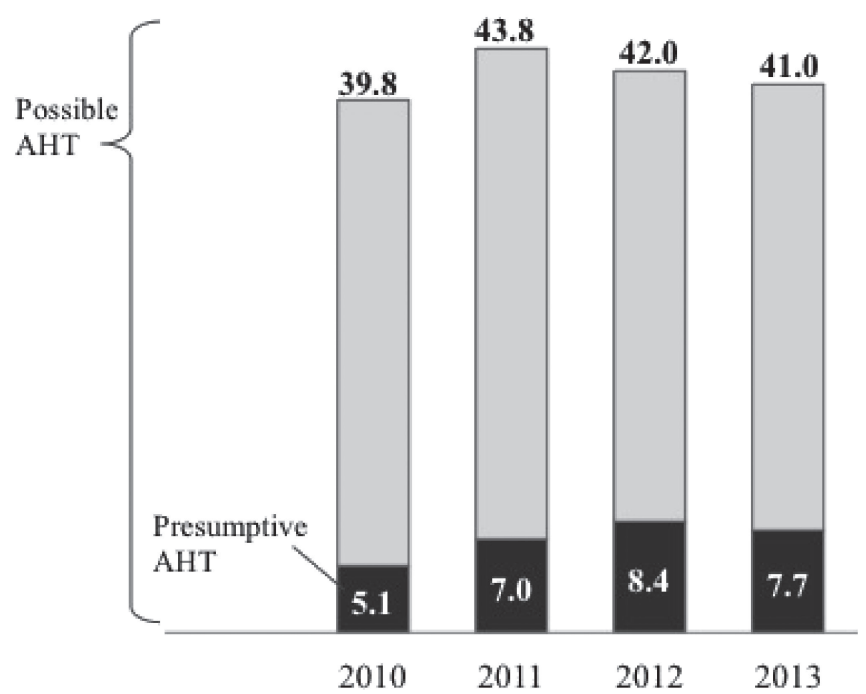

Figure 1. Incidence of presumptive and possible abusive head trauma (AHT) per 100,000 infants between 2010 and 2013 through 2013, Both presumptive and possible AHT had two peaks, at 1-3 and 7-9 months old.

\section{Incidence of presumptive AHT}

The incidence of presumptive AHT was comparatively lower than that of previous studies, which was 13.0-33.4 per 100,000 infants based on ICD-9 or 10 codes in other counties. ${ }^{4,5,8-10,18,27}$ The definitions of AHT to estimate incidences varied among studies. The passive surveillance to capture AHT cases from claim data was obtained from the hospital discharge records used in several studies. ${ }^{5,9,10,27}$ Berger et al reported a high accuracy of the CDC operational definition of AHT using ICD-9CM codes, compared with AHT defined by child protection teams. ${ }^{28}$ However, Japanese coders may not have sufficient training in coding for cause of intentional injury, such as physical abuse (T74.1, T74.8, T74.9), assault (Y00, Y01, Y04, Y08, Y09), or other maltreatment syndromes (Y07.0, Y07.1, Y07.2, Y07.3, Y07.8, Y07.9) that were used in other studies. ${ }^{5,9,10,27}$ Therefore, the insufficient coding for intentional injury might lead to underestimate the incidence of presumptive AHT in Japan. On the other hand, the incidence of possible AHT in this study indicated the possibility of AHT cases at most, because we only could obtain AHT 
Table 4. Characteristics of young children under 36 months old admitted with presumptive and possible abusive head trauma (AHT)

\begin{tabular}{|c|c|c|c|c|c|}
\hline & & \multicolumn{2}{|c|}{$\begin{array}{l}\text { Presumptive AHT } \\
(n=324)\end{array}$} & \multicolumn{2}{|c|}{$\begin{array}{l}\text { Possible AHT } \\
(n=2,603)\end{array}$} \\
\hline & & $n$ & $\%$ & $n$ & $\%$ \\
\hline \multirow[t]{2}{*}{ Sex } & Male & 200 & $61.7 \%$ & 1,553 & $59.7 \%$ \\
\hline & Female & 124 & $38.3 \%$ & 1,050 & $40.3 \%$ \\
\hline \multirow[t]{3}{*}{ Age, years } & 0 & 246 & $75.9 \%$ & 1,425 & $54.7 \%$ \\
\hline & 1 & 43 & $13.3 \%$ & 544 & $20.9 \%$ \\
\hline & 2 & 35 & $10.8 \%$ & 634 & $24.4 \%$ \\
\hline \multirow{4}{*}{$\begin{array}{l}\text { Japan Coma Scale } \\
\text { at admission }\end{array}$} & 0 & 217 & $67.0 \%$ & 1,783 & $68.5 \%$ \\
\hline & 1 & 57 & $17.6 \%$ & 531 & $20.4 \%$ \\
\hline & 2 & 38 & $11.7 \%$ & 245 & $9.4 \%$ \\
\hline & 3 & 12 & $3.7 \%$ & 44 & $1.7 \%$ \\
\hline Duration, days & $\begin{array}{l}\text { Median, IQR } \\
\text { (range) }\end{array}$ & $\begin{array}{l}15 \\
(2-328)\end{array}$ & $7-29$ & $\begin{array}{l}4 \\
(2-752)\end{array}$ & $2-10$ \\
\hline \multirow{4}{*}{$\begin{array}{l}\text { Transfer from } \\
\text { other hospital } \\
\text { Ambulance }\end{array}$} & No & 198 & $61.3 \%$ & 1,811 & $69.7 \%$ \\
\hline & Yes & 125 & $38.7 \%$ & 786 & $30.3 \%$ \\
\hline & No & 191 & $59.1 \%$ & 1,320 & $50.8 \%$ \\
\hline & Yes & 132 & $40.9 \%$ & 1,277 & $49.2 \%$ \\
\hline \multirow[t]{2}{*}{ Poor outcome ${ }^{a}$} & No & 281 & $91.2 \%$ & 2,447 & $96.0 \%$ \\
\hline & Yes & 27 & $8.8 \%$ & 103 & $4.0 \%$ \\
\hline \multirow[t]{2}{*}{ Epidural hemorrhage } & No & 302 & $93.2 \%$ & 2,114 & $81.2 \%$ \\
\hline & Yes & 22 & $6.8 \%$ & 489 & $18.8 \%$ \\
\hline \multirow{3}{*}{$\begin{array}{l}\text { Total admission cost } \\
(1,000 \text { yen } \risingdotseq 10 \mathrm{USD})\end{array}$} & Median, IQR & 754 & 373-1929 & 240 & $119-582$ \\
\hline & Mean, SD & 1,468 & 2007 & 699 & 1372 \\
\hline & (range) & \multicolumn{2}{|c|}{$(6.3-17,400)$} & \multicolumn{2}{|c|}{$(1.5-17,400)$} \\
\hline
\end{tabular}

IQR, interquartile range.

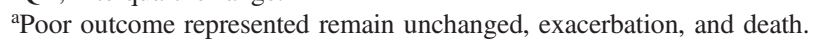

without unintentional injury code. It might include some intracranial injuries that physicians or coders did not code for the cause of injury. Therefore, to obtain accurate incidence for AHT and to establish effective passive surveillance, sufficient education for physicians and coders is needed to ensure the effective evaluation of AHT.
Another method to obtain better estimates for AHT is to utilize active surveillance with multiple sources of information. ${ }^{4,8,18}$ For example, Keenan et $\mathrm{al}^{4}$ abstracted information from medical charts and medical examiner records, reviewed radiological examinations, and utilized determination of AHT by social service agency. Because AHT is a rare event and a large population or multiple years of followup is needed to assess its incidence, conducting active surveillance becomes expensive and time-consuming. Previous studies using passive surveillance showed similar incidence of AHT with that of active surveillance. ${ }^{9,10}$ Therefore, improving better passive surveillance is epidemiologically essential to assess the incidence of AHT. From a practitioner's point of view, detailed information from medical records and child protection services would be helpful for better understanding and prevention of AHT. There are both needs to establish passive and active surveillances that capture the occurrence of AHT in hospitals across the country and share information from multidisciplinary organizations.

\section{Characteristics of presumptive AHT}

This study revealed that the children with presumptive AHT were younger, had severe medical conditions at hospitalization and at discharge, EDH was less frequent, and higher admission cost than possible AHT cases. Longer hospitalization, ${ }^{3}$ poor outcomes, ${ }^{3,29}$ and higher admission $\operatorname{cost}^{30}$ have also been reported as characteristics of AHT. In terms of clinical presentations, seizures at presentation or within 24 hours, and apnea at presentation were significant characteristics of AHT that could lead to a severe JCS score ${ }^{31}$ as seen in presumptive AHT in our study. Therefore, we can confirm that our study subjects had similar clinical severities to that of presumptive AHT cases, and not possible AHT, in the other studies.

\section{The distribution of age in months}

We found two peaks for both presumptive and possible AHT, at

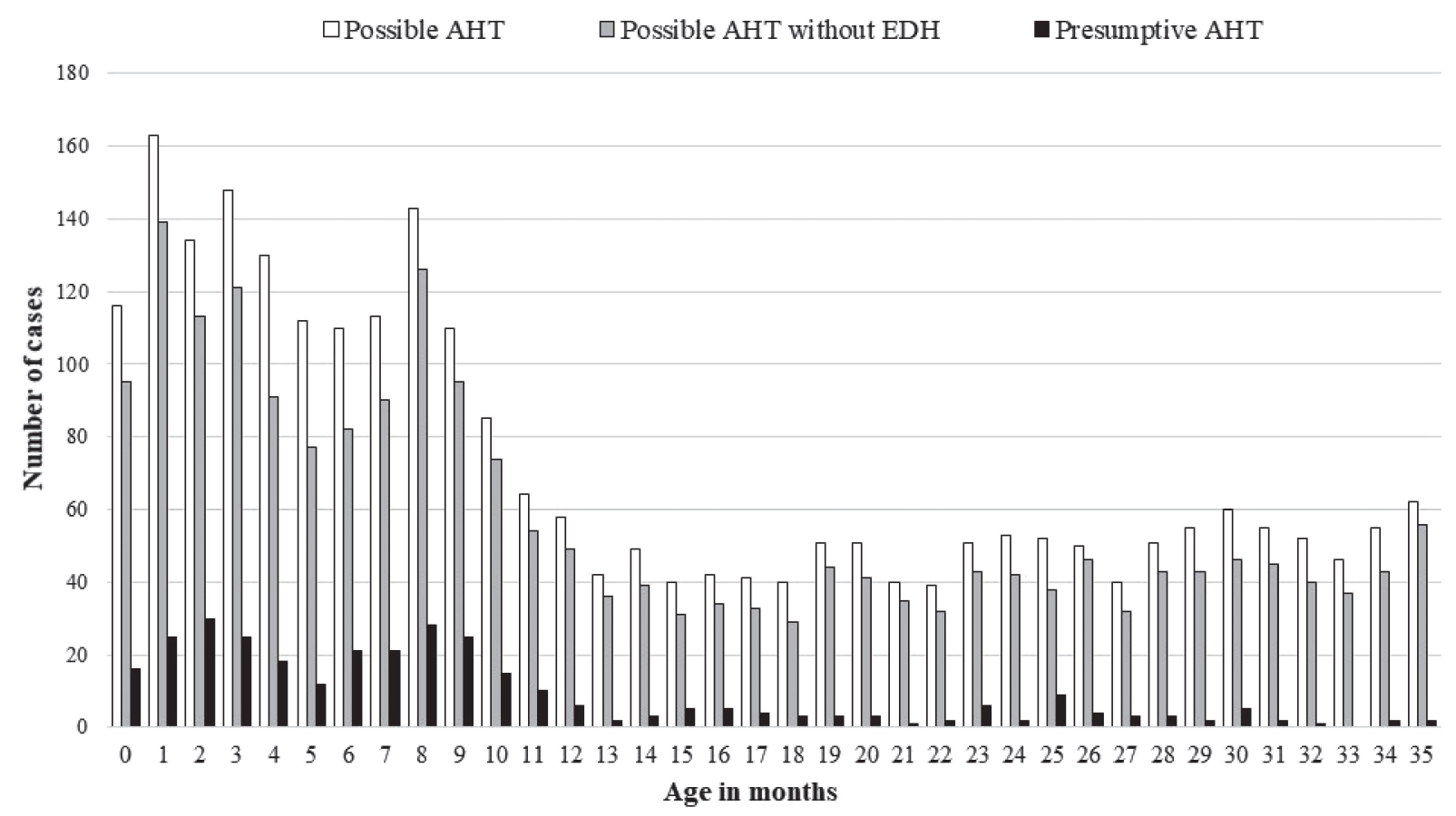

Figure 2. The distribution of age in months for presumptive abusive head trauma (AHT), possible AHT without epidural hemorrhage $(\mathrm{EDH})$, and possible AHT 
1-3 and 7-9 months old. The second-peak distribution in 7-9 months was similar to that of possible AHT without EDH, suggesting that both presumptive and possible AHT during 7-9 months may not have been caused by direct impact, such as falls. A plausible explanation for the first peak at 2 months for presumptive and possible AHT is "the crying period", which is same as in previous studies. ${ }^{15,32}$ The possible interpretations for the second peak at 8 months for presumptive AHT need further consideration. This second peak has not been reported in other countries, and is consistent with a previous study conducted by identifying AHT using CT in Japan. ${ }^{14}$ Other mechanisms need to be considered for presumptive AHT in late infancy. One possible explanation is that different types of parenting stress or challenges occur in the later months of infancy, for example, the transition from milk to solid food ${ }^{33}$; the increased supervision needed as a result of developmental milestones, such as crawling or pulling $\mathrm{up}^{34}$; or anxiety in regard to the delay of those development milestones. Even though Japan has easy access to radiographic examination, as it has the highest rates of CT and MRI in developed countries as well as universal health insurance coverage, the second peak at 8 months for AHT is unique and its underlying mechanisms remain unclear. Further research is needed to target presumptive AHT, especially in the later months of infancy, to determine the possible reasons and preventive strategies in Japan.

\section{Limitations and future direction}

There are several limitations and challenges for further research. First, we utilized the DPC database, which covered more than half of all general hospital beds nationwide. All university hospitals and all 34 children's hospitals and related institutions that provide specialized comprehensive pediatric care were included. We might, however, have missed AHT cases admitted to general hospitals, cases that died before transfer to pediatric tertiary hospitals, or cases that died at home. Moreover, we could not distinguish the children who were admitted or who had transferred hospitals over the years, although we can track the children who were admitted multiple times in the same year. Therefore, future research should utilize the national database for claim records and track patients over years and between hospitals. Second, the incidence of presumptive AHT in this study may have been underestimated due to poor training for physicians and coders to code intentional injury. A multidisciplinary approach for information integration may help to accurately capture the cause of injury in DPC data. Lastly, this study examined the incidence of AHT in population-based samples obtained from the DPC database. However, the DPC database did not include patients who died in the emergency department without being admitted to the hospital. There is a possibility that fatal presumptive AHT cases are underestimated. Information from forensic autopsy records must be added to include fatal AHT cases to determine a more accurate incidence in the future.

\section{Conclusion}

This is the first study to report the incidence among hospitalized children with presumptive and possible AHT under 12 months old in Japan, which was 7.2 and 41.7 per 100,000 infants, respectively. Two peaks for distributions of age in months among children under 36 months old were found at 1-3 and 7-9 months old, a finding which is unique to the Japanese population. Further active surveillance is needed to validate the accuracy of passive surveillance using DPC data. In the meantime, DPC data may be useful to evaluate prevention strategies against AHT in Japan.

\section{ACKNOWLEDGEMENTS}

This study was partially supported by grants from the Research Institute of Science and Technology for Society and the Japan Science and Technology Agency. Dr. Yamaoka was financially supported by the Nippon Foundation International Fellowship program.

Conflicts of interest: None declared.

\section{REFERENCES}

1. King WJ, MacKay M, Sirnick A; Canadian Shaken Baby Study Group. Shaken baby syndrome in Canada: clinical characteristics and outcomes of hospital cases. CMAJ. 2003;168(2):155-159.

2. Starling SP, Holden JR, Jenny C. Abusive head trauma: the relationship of perpetrators to their victims. Pediatrics. 1995;95(2):259262.

3. Lind K, Toure H, Brugel D, Meyer P, Laurent-Vannier A, Chevignard M. Extended follow-up of neurological, cognitive, behavioral and academic outcomes after severe abusive head trauma. Child Abuse Negl. 2016;51:358-367.

4. Keenan HT, Runyan DK, Marshall SW, Nocera MA, Merten DF, Sinal SH. A population-based study of inflicted traumatic brain injury in young children. JAMA. 2003;290(5):621-626.

5. Shanahan ME, Zolotor AJ, Parrish JW, Barr RG, Runyan DK. National, regional, and state abusive head trauma: application of the CDC algorithm. Pediatrics. 2013;132(6):e1546-e1553.

6. Hobbs C, Childs AM, Wynne J, Livingston J, Seal A. Subdural haematoma and effusion in infancy: an epidemiological study. Arch Dis Child. 2005;90(9):952-955.

7. Liley W, Stephens A, Kaltner M, et al. Infant abusive head trauma incidence, outcomes and awareness. Aust Fam Physician. 2012; 41(10):823-826.

8. Kelly P, Farrant B. Shaken baby syndrome in New Zealand, 2000 2002. J Paediatr Child Health. 2008;44(3):99-107.

9. Fujiwara T, Barr RG, Brant RF, Rajabali F, Pike I. Using International Classification of Diseases, 10th edition, codes to estimate abusive head trauma in children. Am J Prev Med. 2012;43(2):215220.

10. Ellingson KD, Leventhal JM, Weiss HB. Using hospital discharge data to track inflicted traumatic brain injury. Am J Prev Med. 2008; 34(4)(Suppl):S157-S162.

11. Fujiwara T, Yamaoka Y, Morisaki N. Self-reported prevalence and risk factors for shaking and smothering among mothers of 4-monthold infants in Japan. J Epidemiol. 2016;26(1):4-13.

12. Ikegami N, Yoo BK, Hashimoto H, et al. Japanese universal health coverage: evolution, achievements, and challenges. Lancet. 2011; 378(9796):1106-1115.

13. OECD. Medical technologies. OECD Publishing Health at a Glance 2015: OECD Indicators Web site. http://www.oecd-ilibrary.org/sites/ health_glance-2015-en/06/02/index.html?itemId=/content/chapter/ health_glance-2015-31-en\&mimeType=text/html. Published 2015. Accessed.

14. Fujiwara T, Okuyama M, Miyasaka M. Characteristics that distinguish abusive from nonabusive head trauma among young children who underwent head computed tomography in Japan. Pediatrics. 2008;122(4):e841-e847.

15. Parks SE, Kegler SR, Annest JL, Mercy JA. Characteristics of fatal abusive head trauma among children in the USA: 2003-2007: an application of the CDC operational case definition to national vital statistics data. Inj Prev. 2012;18(3):193-199.

16. Barr RG, Trent RB, Cross J. Age-related incidence curve of hospitalized Shaken Baby Syndrome cases: convergent evidence for crying as a trigger to shaking. Child Abuse Negl. 2006;30(1):7-16.

17. Selassie AW, Borg K, Busch C, Russell WS. Abusive head trauma 
in young children: a population-based study. J Trauma Nurs. 2014; 21(2):72-82.

18. Barlow KM, Minns RA. Annual incidence of shaken impact syndrome in young children. Lancet. 2000;356(9241):1571-1572.

19. Xia X, Xiang J, Shao J, et al. Characteristics and trends of hospitalized pediatric abuse head trauma in Wuhan, China: 20022011. Int J Environ Res Public Health. 2012;9(11):4187-4196.

20. Yasunaga H, Matsui H, Horiguchi H, Fushimi K, Matsuda S. Clinical epidemiology and health services research using the diagnosis procedure combination database in Japan. Asian Pacific J Dis Manage. 2013;7(1-2):19-24.

21. Health Statistics Office VaHSD, Statistics and Information Department, Minister's Secretariat. Surveys of Medical Institutions. Table J2. FY2014. http://www.e-stat.go.jp/SG1/estat/List.do?lid= 000001141080. Published 2015. Accessed 11/22, 2016.

22. Yamana H, Moriwaki M, Horiguchi H, Kodan M, Fushimi K, Yasunaga $\mathrm{H}$. Validity of diagnoses, procedures, and laboratory data in Japanese administrative data. J Epidemiol. 2017;27(10):476-482.

23. Yamana H, Matsui H, Fushimi K, Yasunaga H. Procedure-based severity index for inpatients: development and validation using administrative database. BMC Health Serv Res. 2015;15:261.

24. Sumitani M, Uchida K, Yasunaga H, et al. Prevalence of malignant hyperthermia and relationship with anesthetics in Japan: data from the diagnosis procedure combination database. Anesthesiology. 2011;114(1):84-90.

25. Parks S, Annest J, Hill H, Karch D. Pediatric Abusive Head Trauma: Recommended Definitions for Public Health Surveillance and Research. Centers for Disease Control and Prevention. https:// www.cdc.gov/violenceprevention/pdf/pedheadtrauma-a.pdf. Published 2012. Accessed 11/22, 2016.
26. Irie F, Le Brocque R, Kenardy J, Bellamy N, Tetsworth K, Pollard C. Epidemiology of traumatic epidural hematoma in young age. J Trauma. 2011;71(4):847-853.

27. Parks S, Sugerman D, Xu L, Coronado V. Characteristics of nonfatal abusive head trauma among children in the USA, 2003-2008: application of the CDC operational case definition to national hospital inpatient data. Inj Prev. 2012;18(6):392-398.

28. Berger RP, Parks S, Fromkin J, Rubin P, Pecora PJ. Assessing the accuracy of the International Classification of Diseases codes to identify abusive head trauma: a feasibility study. Inj Prev. 2015; 21(e1):e133-e137.

29. Chevignard MP, Lind K. Long-term outcome of abusive head trauma. Pediatr Radiol. 2014;44(Suppl 4):S548-S558.

30. Miller TR, Steinbeigle R, Lawrence BA, et al. Lifetime cost of abusive head trauma at ages 0-4, USA. Prev Sci. 2018;19(6):695704.

31. Piteau SJ, Ward MG, Barrowman NJ, Plint AC. Clinical and radiographic characteristics associated with abusive and nonabusive head trauma: a systematic review. Pediatrics. 2012;130(2):315-323.

32. Lee C, Barr RG, Catherine N, Wicks A. Age-related incidence of publicly reported shaken baby syndrome cases: is crying a trigger for shaking? J Dev Behav Pediatr. 2007;28(4):288-293.

33. Equal Employment Children and Families Bureau, Ministry of Health Labour and Welfare. National nutrition survey on preschool children, FY2015. http://www.mhlw.go.jp/stf/seisakunitsuite/ bunya/0000134208.html. Published 2016. Accessed 06/12, 2017.

34. Equal Employment Children and Families Bureau, Welfare. MoHLa. National growth survey on preschool children, FY2010. http://www.mhlw.go.jp/toukei/list/73-22.html. Published 2010. Accessed 06/12, 2017 